\title{
Factor VIII gene $(F 8)$ mutation and risk of inhibitor development in nonsevere hemophilia A
}

\author{
Corien L. Eckhardt, ${ }^{1}$ Alice S. van Velzen, ${ }^{1}$ Marjolein Peters, ${ }^{1}$ Jan Astermark, ${ }^{2}$ Paul P. Brons, ${ }^{3}$ Giancarlo Castaman, ${ }^{4}$ \\ Marjon H. Cnossen, ${ }^{5}$ Natasja Dors, ${ }^{6}$ Carmen Escuriola-Ettingshausen, ${ }^{7}$ Karly Hamulyak, ${ }^{8}$ Daniel P. Hart, ${ }^{9}$ \\ Charles R. M. Hay, ${ }^{10}$ Saturnino Haya, ${ }^{11}$ Waander L. van Heerde, ${ }^{3}$ Cedric Hermans, ${ }^{12}$ Margareta Holmström, ${ }^{13}$ \\ Victor Jimenez-Yuste, ${ }^{14}$ Russell D. Keenan, ${ }^{15}$ Robert Klamroth, ${ }^{16}$ Britta A. P. Laros-van Gorkom, ${ }^{3}$ \\ Frank W. G. Leebeek, ${ }^{5}$ Ri Liesner, ${ }^{17}$ Anne Mäkipernaa, ${ }^{18}$ Christoph Male, ${ }^{19}$ Evelien Mauser-Bunschoten, ${ }^{20}$ \\ Maria G. Mazzucconi, ${ }^{21}$ Simon McRae, ${ }^{22}$ Karina Meijer, ${ }^{23}$ Michael Mitchell, ${ }^{24}$ Massimo Morfini, ${ }^{25}$ Marten Nijziel, ${ }^{26}$ \\ Johannes Oldenburg, ${ }^{27}$ Kathelijne Peerlinck, ${ }^{28}$ Pia Petrini, ${ }^{13}$ Helena Platokouki, ${ }^{29}$ Sylvia E. Reitter-Pfoertner, ${ }^{19}$ \\ Elena Santagostino, ${ }^{30}$ Piercarla Schinco, ${ }^{31}$ Frans J. Smiers, ${ }^{32}$ Berthold Siegmund, ${ }^{33}$ Annarita Tagliaferri, ${ }^{34}$ Thynn T. Yee, ${ }^{35}$ \\ Pieter Willem Kamphuisen, ${ }^{1}$ Johanna G. van der Bom, ${ }^{32,36}$ and Karin Fijnvandraat, ${ }^{1}$ for the INSIGHT Study Group
}

\begin{abstract}
${ }^{1}$ Academic Medical Center, Amsterdam, The Netherlands; ${ }^{2}$ Skåne University Hospital, Malmö, Sweden; ${ }^{3}$ Radboud University Nijmegen Medical Centre, Nijmegen, The Netherlands; ${ }^{4}$ San Bortolo Hospital, Vicenza, Italy; ${ }^{5}$ Erasmus University Medical Center, Rotterdam, The Netherlands; ${ }^{6} \mathrm{Catharina}$ Hospital, Eindhoven, The Netherlands; ${ }^{7} \mathrm{JW}$ Goethe University Hospital, Frankfurt, Germany; ${ }^{8}$ Maastricht University Medical Centre, Maastricht, The Netherlands; ${ }^{9}$ Royal London Hospital, Barts and The London School of Medicine and Dentistry, London, United Kingdom; ${ }^{10}$ Manchester Royal Infirmary, Manchester, United Kingdom; ${ }^{11}$ University Hospital la Fe, Valencia, Spain; ${ }^{12}$ Saint-Luc University Hospital, Brussels, Belgium; ${ }^{13}$ Karolinska University Hospital, Stockholm, Sweden; ${ }^{14}$ University Hospital La Paz and Autonoma University, Madrid, Spain; ${ }^{15}$ Alderhey Children's Hospital, Liverpool, United Kingdom; ${ }^{16}$ Vivantes Klinikum im Friedrichshain, Berlin, Germany; ${ }^{17}$ Great Ormond Street National Health Service Trust, London, United Kingdom; ${ }^{18}$ Children's Hospital, Helsinki University Central Hospital, Helsinki, Finland; ${ }^{19}$ Medical University of Vienna, Vienna, Austria; ${ }^{20}$ University Medical Center Utrecht, Utrecht, The Netherlands; ${ }^{21}$ Sapienza University of Rome, Rome, Italy; ${ }^{22}$ Royal Adelaide Hospital, Adelaide, Australia; ${ }^{23}$ University Medical Center Groningen, Groningen, The Netherlands; ${ }^{24}$ Guy's and St. Thomas' National Health Service Foundation Trust, London, United Kingdom; ${ }^{25} \mathrm{Azienda}$ University Hospital Careggi, Florence, Italy; ${ }^{26}$ Maxima Medical Center, Eindhoven/Veldhoven, The Netherlands; ${ }^{27}$ University Clinic of Bonn, Bonn, Germany; ${ }^{28}$ University of Leuven, Leuven, Belgium; ${ }^{29}$ Aghia Sofia Children's Hospital, Athens, Greece; ${ }^{30}$ Ospedale Maggiore Policlinico, Fondazione Istituto Di Ricovero e Cura a Carattere Scientifico Ca' Granda, Milan, Italy; ${ }^{31}$ San Giovanni Battista "Molinette" Hospital, Turin, Italy; ${ }^{32}$ Leiden University Hospital, Leiden, The Netherlands; ${ }^{33}$ Raphaelsklinik, Munster, Germany; ${ }^{34}$ University Hospital of Parma, Parma, Italy; ${ }^{35}$ Royal Free Hospital, London, United Kingdom; and ${ }^{36}$ Sanquin Research, Leiden, The Netherlands
\end{abstract}

\section{Key Points}

- The inhibitor incidence in nonsevere hemophilia A patients with certain F8 mutations approaches the inhibitor incidence in severe patients.

- These findings are highly relevant for clinical practice, as they facilitate identification of high-risk patients based on F8 genotype.

Neutralizing antibodies (inhibitors) toward factor VIII form a severe complication in nonsevere hemophilia $A$, profoundly aggravating the bleeding pattern. Identification of high-risk patients is hampered by lack of data that take exposure days to therapeutic factor VIII concentrates into account. In the INSIGHT study, we analyzed the association between $F 8$ mutation and inhibitor development in patients with nonsevere hemophilia A (factor VIII 2-40 IU/dL). This analysis included 1112 nonsevere hemophilia A patients from $\mathbf{1 4}$ centers in Europe and Australia that had genotyped at least $70 \%$ of their patients. Inhibitor risk was calculated as KaplanMeier incidence with cumulative number of exposure days as the time variable. During 44800 exposure days (median, 24 exposure days per patient; interquartile range [IQR], 7-90), 59 of the 1112 patients developed an inhibitor; cumulative incidence of $5.3 \%(95 \%$ confidence interval $[\mathrm{Cl}], 4.0-6.6)$ after a median of 28 exposure days (IQR, 12-71). The inhibitor risk at 50 exposure days was $6.7 \%(95 \% \mathrm{Cl}, 4.5-8.9)$ and at 100 exposure days the risk further increased to $13.3 \%(95 \% \mathrm{Cl}, 9.6-17.0)$. Among a total of 214 different $F 8$ missense mutations 19 were associated with inhibitor development. These results emphasize the importance of $F 8$ genotyping in nonsevere hemophilia A. (Blood. 2013; 122(11):1954-1962)

\section{Introduction}

Patients with hemophilia A who are treated with factor VIII concentrates are at risk of developing factor VIII neutralizing alloantibodies (inhibitors). ${ }^{1,2}$ Inhibitor development is one of

Submitted February 16, 2013; accepted June 28, 2013. Prepublished online as Blood First Edition paper, August 7, 2013; DOI 10.1182/blood-2013-02483263.

The online version of the article contains a data supplement. the most challenging complications in the treatment of hemophilia A, as it increases the bleeding tendency while it renders treatment with therapeutic factor VIII concentrates ineffective.

The publication costs of this article were defrayed in part by page charge payment. Therefore, and solely to indicate this fact, this article is hereby marked "advertisement" in accordance with 18 USC section 1734. 


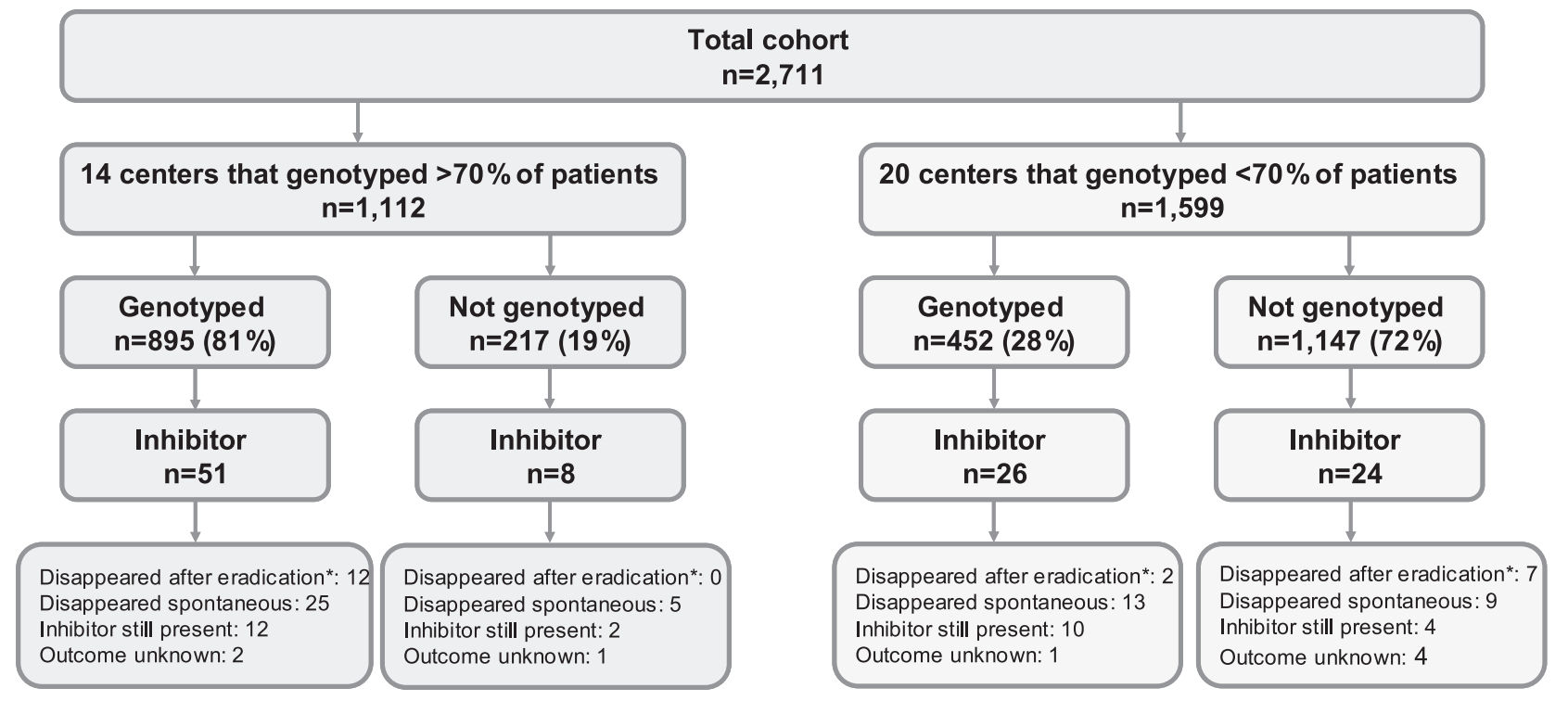

Figure 1. Flowchart of patients included in the study. ${ }^{*}$ Eradication treatment: ITI, immunodulating treatment or a combination of these.

Treatment of patients with inhibitors can be very complicated and costly. ${ }^{3}$

Although inhibitor development is less frequently observed in patients with nonsevere hemophilia A (baseline factor VIII activity of 2-40 IU/dL), the clinical impact can be profound. In these patients, inhibitors may also interact with their endogenous factor VIII, resulting in a decrease of the factor VIII plasma level below $1 \mathrm{IU} / \mathrm{dL}^{1}$ and major bleeding complications. ${ }^{4}$ Identification of patients at risk of developing inhibitors may help to prevent this serious complication. However, currently there are no tools available to predict individual inhibitor risk in nonsevere hemophilia patients.

The type of mutation in the factor VIII gene $(F 8)$ is an important risk factor for inhibitor development. ${ }^{5-7}$ Nonsevere hemophilia A is generally caused by $F 8$ missense mutations. ${ }^{8}$ Despite information on large numbers of $F 8$ mutations associated with nonsevere hemophilia A that is collected in international databases, ${ }^{9,10}$ it is not possible to calculate the inhibitor risk for specific $F 8$ mutations, as data on exposure days to therapeutic factor VIII concentrates are lacking. Moreover, there may be a reporting bias in these databases favoring registration of those with identified inhibitors.

As inhibitor development is elicited by the exposure to therapeutic factor VIII, the risk of developing inhibitors strongly depends on the cumulative number of exposure days. Information regarding exposure to factor VIII concentrates is especially important in nonsevere hemophilia A patients, as they receive factor VIII replacement therapy on an irregular basis and much less frequently than severe hemophilia A patients on account of having milder bleeding phenotypes. Previous studies in severe hemophilia A demonstrate the risk of developing inhibitors after more than 50 exposure days decreases to less than $1 \%{ }^{1}$ The majority of patients with severe hemophilia A receives 50 exposure days within the first few years of life. However, in nonsevere hemophilia A, even some adult patients may still have less than 50 exposure days to therapeutic factor VIII and will thus still be at risk of developing inhibitors.

This study includes the largest cohort of patients with nonsevere hemophilia A to assess the risk of inhibitor development. It is the first analysis of the association between inhibitor development and F8 mutation in nonsevere hemophilia that takes cumulative exposure to therapeutic factor VIII concentrates into account. This provides valuable data enabling a more personalized inhibitor risk estimation, which is of immediate clinical relevance. Moreover, it may improve our understanding of alloreactivity in response to an intravenous protein therapeutic.

\section{Patients and methods}

\section{Subjects and study design}

This retrospective cohort study includes patients from a source population consisting of all patients $(\mathrm{n}=2711)$ with nonsevere hemophilia A (baseline factor VIII activity of 2-40 IU/dL) who received at least one exposure to factor VIII concentrate between January 1, 1980 (when factor VIII concentrates became more widely available) and January 1, 2011 in 1 of the 34 participating hemophilia treatment centers of the INSIGHT study. Participating centers (listed in the data supplement on the Blood Web site.) were located in 10 European countries and Australia. The institutional review boards of all participating centers approved the study and have indicated that signed informed consent was not required. All patients from the source population were followed-up from birth until death, emigration, loss-to-follow-up, or the end of the study. This study was conducted in accordance with the Declaration of Helsinki.

Because inhibitor patients may be more likely to be genotyped, we were concerned that inhibitor patients would be overrepresented in our cohort. To reduce the risk of selection bias, we restricted our analysis to all patients $(n=1112)$ of the centers that had genotyped at least $70 \%$ of their patients (see flowchart of patients in Figure 1). The cutoff of $\geqslant 70 \%$ was arbitrarily chosen before initial analysis. In these 14 centers, which are located in 8 countries, patients were genotyped as part of the routine practice, independent of patient characteristics. Patients were included in the center where they received treatment with factor VIII concentrates (prior to inhibitor development). Patients who were referred to a participating center once they had already developed an inhibitor were excluded from the analysis.

\section{Data collection}

Data were collected from medical records using a standardized electronic case report form. The following data on patient characteristics were collected: date of birth, ethnicity, $F 8$ mutation, family history of hemophilia and inhibitors. Additionally, we collected data on the total number of days on which the patient was exposed to therapeutic factor VIII concentrates before inhibitor development or the end of the follow-up period. An exposure day 
was defined as a calendar day during which 1 or more infusions of factor VIII concentrates were administered. The cumulative number of exposure days was classified into the following 7 categories: 1 to 5,5 to 10,10 to 20,20 to 30,30 to 50,50 to 100 , and $\geqslant 100$ exposure days. Detailed information for each exposure day (such as date, factor VIII product type, and reason for treatment) were not necessary for the analysis of the association between inhibitor development and $F 8$ mutation, and were not collected due to feasibility reasons.

\section{Laboratory tests and F8 mutation analysis}

Results of the following laboratory tests were collected to confirm the diagnosis of nonsevere hemophilia A: baseline factor VIII activity (FVIII:C, assessed by the 1-stage clotting factor VIII assay, 2-40 IU/dL), von Willebrand factor antigen level (VWF:Ag), and von Willebrand factor activity (VWF:RCo). Patients with FVIII:C levels $<2$ IU/dL were not included in the study to avoid misclassification and inclusion of severe hemophilia A patients because older FVIII:C tests were less precise in measuring the lower ranges of FVIII:C. $F 8$ mutation was collected from authorized genetic laboratory reports in the patient's records or derived from his pedigree (in 5.3\% of the patients) when the causative mutation had been identified in another family member. Standard methods for the analysis of the F8 were used at each individual laboratory as previously described. ${ }^{11}$ Each mutation was reviewed and identified using the traditional nomenclature based on the mature processed protein. Amino acid numbering for point mutations is given for the mature processed protein, as universally used in $F 8$ studies. To convert to Human Genome Variation Society (HGVS) type of numbering, add 19 to positive numbers. ${ }^{12}$

\section{Definition of inhibitor development}

Inhibitor tests were done at the discretion of each center, and the results of all inhibitor tests were reviewed to confirm the inhibitor status of the patients (no data on the number of inhibitor tests performed on each patient were available). Inhibitory antibodies were quantified at each local laboratory by the original Bethesda method or the Nijmegen modified assay from the 1990s onwards. ${ }^{13,14}$

Only patients with a clinically relevant inhibitor were classified to be positive for an inhibitor in the analysis. A clinically relevant inhibitor was defined as having at least 2 positive Bethesda inhibitor assay titers of $\geqslant 1.0$ Bethesda inhibitor assay unit per $\mathrm{mL}(\mathrm{BU} / \mathrm{mL})$. A high titer inhibitor was defined as a historical peak titer $>5.0 \mathrm{BU} / \mathrm{mL}$. Patients with inhibitor titers between 0.6 and $1.0 \mathrm{BU} / \mathrm{mL}$ had to fulfill 1 of the following 2 criteria to be classified as having a clinically relevant inhibitor: a decrease in factor VIII plasma level to at least $50 \%$ of the baseline level, or a reduced half-life after factor VIII administration of less than 6 hours. Patients who were not tested for factor VIII inhibitors during the follow-up period and who had no clinical features of inhibitor development (eg, increased bleeding tendency) were classified as negative for inhibitors $(\mathrm{n}=5)$.

\section{Data analysis}

To adjust for the varying number of cumulative exposure days to factor VIII concentrates, we calculated Kaplan-Meier cumulative inhibitor incidences with inhibitor development as the event, and the cumulative number of exposure days as the time variable. Missing values of cumulative exposure days were imputed using multiple linear regression methods in $4 \%$ of the patients. ${ }^{15}$ For F8 mutations carried by $>10$ subjects, Kaplan-Meier cumulative inhibitor incidences were interpreted as inhibitor risk and are presented as percentages with $95 \%$ confidence interval (CI). To enable comparison of inhibitor risk between $F 8$ mutations at a certain time point in treatment, cumulative inhibitor incidences are given at 20 and 50 exposure days. The risk is calculated by dividing the number of inhibitor patients with a specific mutation by the total number of patients with the same mutation that have a certain number of exposure days (eg, 20 or 50 exposure days). Thus, an inhibitor risk of $30 \%$ after 50 exposure days for a specific mutation means that after 50 exposure days to therapeutic factor VIII concentrates $30 \%$ of the patients with this mutation developed an inhibitor.
Table 1. Characteristics of the total study population $(n=1112)$

\begin{tabular}{|cc|}
\hline Parameter & $\mathbf{n}(\%)$ or median (IQR) \\
\hline Total length of observation, years & 25700 \\
\hline Total length of observation, ED & 44800 \\
Baseline FVIII:C, IU/dL & $11.5(6.0-20.0)$ \\
\hline Classified into FVIII:C categories, IU/dL & \\
\hline $2-5$ & $231(20.8)$ \\
\hline $6-10$ & $283(25.4)$ \\
\hline $11-20$ & $345(31.0)$ \\
\hline $21-30$ & $180(16.2)$ \\
\hline $31-40$ & $73(6.6)$ \\
\hline F8 genotype known & $895(80.5)$ \\
\hline Positive family history of inhibitors & $26(2.3)$ \\
\hline Ethnicity & \\
\hline White & $1,049(94.3)$ \\
\hline African-American & $14(1.3)$ \\
\hline Asian & $18(1.6)$ \\
\hline Other & $24(2.2)$ \\
\hline Unknown & $7(0.6)$ \\
\hline Age at the end of follow-up, years & $38(18-56)$ \\
\hline Number inhibitors & $59(5.3)$ \\
\hline Cumulative ED to factor VIII concentrates & $24(7-90)$ \\
\hline Classified into ED categories: & \\
\hline $1-5$ & $166(14.9)$ \\
\hline $5-10$ & $151(13.6)$ \\
\hline $10-20$ & $200(18.0)$ \\
\hline $20-30$ & $131(11.8)$ \\
\hline $30-50$ & $107(9.6)$ \\
\hline $50-100$ & $156(14.0)$ \\
\hline$\geq 100$ & $179(16.1)$ \\
\hline Unknown & $22(2.0)$ \\
\hline
\end{tabular}

ED, exposure days to factor VIII concentrates.

\section{Results}

\section{Patient characteristics}

In total, 1112 subjects with a confirmed diagnosis of nonsevere hemophilia A received 1 or more exposures to factor VIII concentrate and were included in the study population. Patients who were born between 1908 and 2009 were observed for approximately 25700 patient years and 44800 exposure days with a median follow-up of 38 years (IQR, 18-56) and 24 exposure days (IQR, 7-90) per patient. Two-thirds of patients had fewer than 50 exposure days at the end of follow-up $(n=755[68 \%])$, with most of them $(n=517)$ who had fewer than 20 exposure days. These and other demographic characteristics of the patients were comparable to that of the source population of 2711 patients (shown in Table 1).

\section{Inhibitor development}

Inhibitors developed in 59 patients, cumulative incidence of $5.3 \%$ (95\% CI, 4.0-6.6) at a median age of 46 years (IQR, 18-65). Most inhibitors developed in the period of 2000 to $2010(n=37$ [64\%]), as compared with 17 inhibitors (29\%) between 1990 and 1999 and $4(7 \%)$ between 1980 and 1989. Patients who developed an inhibitor between 1980 and 1989 were younger at the time of inhibitor development (1980-1989: median age at inhibitor development, 25 years [IQR, 19-60]; 1990-1999: median, 52 years [IQR, 21-66]; 2000-2010: median, 47 years [IQR, 15-65]) and had less cumulative exposures to factor VIII concentrates before inhibitor development (1980-1989: median, 11 ED [IQR, 6-75]; 1990-1999: median, 12 ED [IQR, 8-41]; 2000-2010: median, 32 ED [IQR, 


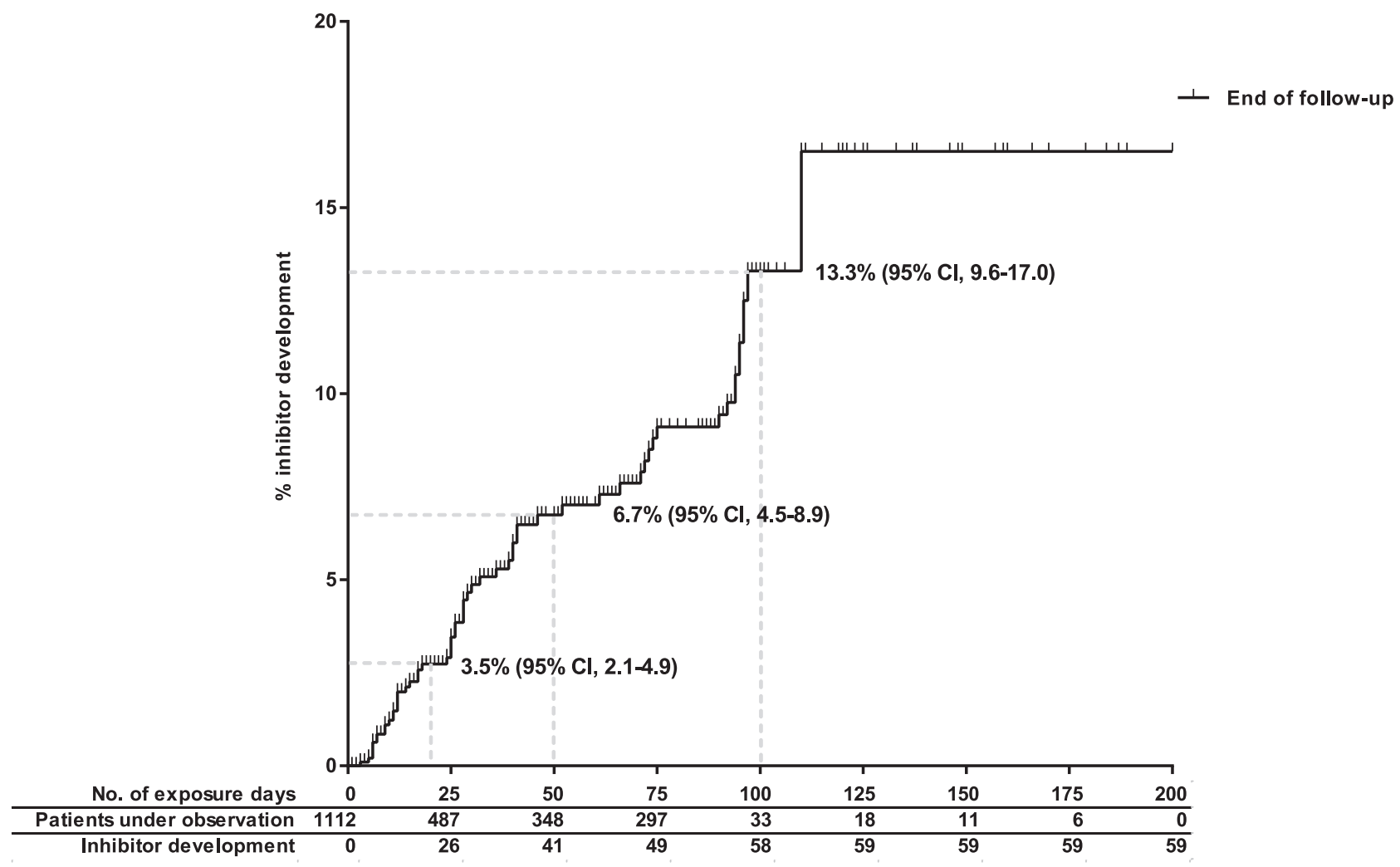

Figure 2. Cumulative inhibitor incidence in 1112 nonsevere hemophiliaA patients, according to cumulative exposure days to factor VIII concentrates.

21-75 ED]). The median peak inhibitor titer was $9 \mathrm{BU} / \mathrm{mL}$ (IQR, $2-30)$ and $36(61 \%)$ patients had a high titer inhibitor. Endogenous FVIII:C was decreased in $34(58 \%)$ inhibitor patients; FVIII:C fell below $\leqslant 2 \mathrm{IU} / \mathrm{dL}$ in $20(34 \%)$ patients, of whom 16 patients had FVIII:C $\leqslant 1$ IU/dL (27\%). At inhibitor detection, 30 (51\%) patients had an increased bleeding tendency and 47 (80\%) patients needed treatment of bleeding when the inhibitor was present. In 11 inhibitor patients $(19 \%)$ there was no clinical sign of inhibitor development at presentation and the inhibitor was detected during routine inhibitor testing. The inhibitor became undetectable in 42 of 59 patients (71\%) after a median of 45 weeks (IQR, 13-108), and in 12 patients $(29 \%)$ after inhibitor eradication therapy (immune tolerance induction $\mathrm{n}=7$, immunosuppressive therapy $\mathrm{n}=4$, both $\mathrm{n}=1)$. In 30 patients $(71 \%)$ the inhibitor cleared spontaneously without specific inhibitor eradication therapy (of whom 18 were rechallenged to factor VIII concentrates after the inhibitor became undetectable), and in 3 patients $(5 \%)$ eradication therapy was unknown. In 14 patients $(15 \%)$ the inhibitor was still present at the end of follow-up, and in 3 patients (5\%) inhibitor status at the end of follow-up was unknown (Figure 1).

Inhibitors were detected after a median of 28 exposure days (IQR, 12-71). Forty-one patients (69\%) developed an inhibitor before 50 exposure days, 17 patients (29\%) between 50 and 100 exposure days, and only 1 patient (2\%) after more than 100 exposure days. The inhibitor risk at 20 and 50 exposure days was 3.5\% (95\% CI, 2.1-4.9) and $6.7 \%$ (95\% CI, 4.5-8.9), respectively. The inhibitor risk was $13.3 \%$ (95\% CI, 9.6-17.0) in patients with 100 exposure days (Figure 2).

Patients who developed inhibitors were comparable to patients who did not develop inhibitors regarding ethnicity (inhibitor patients, 97\% white; noninhibitor patients, $95 \%$ white) and severity of disease (inhibitor patients: median, FVIII:C, $10 \mathrm{IU} / \mathrm{dL}$ [IQR, 6-19]; noninhibitor patients: median, FVIII:C, 10.5 IU/dL [IQR, 6-18]).

\section{F8 mutations}

F8 genotype was known in 895 patients $(81 \%)$ of the study population $(\mathrm{n}=1112)$ and in 51 (51 of 59 [86\%]) patients who had developed an inhibitor. In total, 214 different missense mutations $(\mathrm{n}=865$ patients [78\%]) and 17 other mutations $(\mathrm{n}=30$ patients [3\%]) were identified in F8 (supplemental Table 1). prevalent amino acid substitutions were Arg593Cys (9\%), Arg2150His (5\%), Asn618Ser (5\%), and Arg531Cys (3\%). The complete list of mutations of the centers that had genotyped at least $70 \%$ of their patients $(n=1112)$ and of the total INSIGHT cohort $(n=2711)$ are presented in supplemental Tables 1 and 2 . There was a wide variation of baseline FVIII:C levels within patients carrying the same $F 8$ mutation (supplemental Tables 1 and 2) that could not be explained by interlaboratory variability or variation of FVIII:C assays over time. There was a variation in inhibitor risk between FVIII:C levels, however, there was no trend observed toward patients with lower FVIII:C levels having the highest inhibitor risk (data not shown).

\section{Association between inhibitors and F8 mutation}

Nineteen mutations (Leu412Phe, Arg531Cys, Arg593Cys, Asn618Ser, Pro1761Gln, Phe1775Val, Arg1781Gly, Pro1854Leu, Arg1997Trp, Asp2074Gly, Phe2101Cys, Tyr2105Cys, Arg2150His, Arg2159Cys, Glu2228Asp, Trp2229Cys, Val2232Ala, His2309Asp, Stop2333Cys) were associated with inhibitor development and were all located in the A2 domain of the heavy chain and in the A3, C1, and C2 domains of the light chain of the factor VIII protein (Figure 3). These mutations were all missense mutations in $F 8$ and were identified in a total of 333 patients including the 51 genotyped patients with inhibitors. Table 2 and supplemental Table 1 summarize the numbers and proportions of patients with inhibitors according to the $F 8$ mutation. Inhibitor risk for individual $F 8$ mutations varied 
between $0 \%$ to $42 \%$ at 50 exposure days (Table 2). Two of the $19 F 8$ mutations associated with inhibitor development (Phe1775Val, Stop2333Cys) have not been reported before in the the Haemophilia A Mutation, Structure, Test and Resource site or Center for Disease Control Hemophilia A Mutation Project database and five F8 mutations were not previously reported with inhibitor development (Leu412Phe, Arg1781Gly, Phe2101Cys, Arg2159Cys, His2309Asp). ${ }^{9,10}$

\section{Discussion}

This is the largest cohort of nonsevere hemophilia A patients in which the inhibitor risk and its association with $F 8$ mutations has been assessed, taking exposure days into account. The inhibitor risk was $6.7 \%$ at 50 exposure days, rising to $13.3 \%$ at 100 exposure days. We identified 19 mutations associated with inhibitor development (of which 7 were not previously reported with inhibitors)- from a total of 214 different missense mutations. ${ }^{9,10}$ This information emphasizes the importance of $F 8$ genotyping in nonsevere hemophilia A patients. It also demonstrates the importance of reporting inhibitor occurrence to either of the international databases if these are to be an important ongoing resource for inhibitor risk assessment. ${ }^{16}$

Importantly, the risk of inhibitor development in patients with nonsevere hemophilia approaches the risk of severe hemophilia patients when inhibitor development is evaluated as a function of exposure to factor VIII concentrates. ${ }^{1,17}$ This highlights the substantial risk of inhibitor development in nonsevere hemophilia A patients that has been previously underestimated. Given that unexposed patients were excluded from the study population, and that a large proportion of patients $(68 \%)$ included in the study had fewer than 50 exposure days, there is a large population of unchallenged patients who may need treatment with factor VIII concentrates in the future. Thus, inhibitor development in nonsevere hemophilia A patients may become an important future burden of care in hemophilia treatment.

As the inhibitor risk of several missense mutations approaches that of severe patients, this may suggest that these single amino acid mutations are highly immunogenic. Previous observations suggest that both position and type of substitution of missense mutations may influence the inhibitor risk. Consistent with these observations, the mutations associated with inhibitors in the INSIGHT cohort were all located within the regions encoding for the light chain and the A2 domain of factor VIII. ${ }^{8}$ A recent study by Schwaab et al ${ }^{18}$ indicates that the risk of inhibitor formation is significantly higher if the substituted amino acid in the factor VIII protein belongs to another physicochemical class than the original residue. Our study was epidemiological in nature and did not aim to elucidate the immunologic mechanism underlying the association of the $F 8$ gene mutation and inhibitor risk. This will definitely be an important aim for future studies in this field. There was a wide variation in inhibitor risk between patients with different FVIII:C levels and no trend was observed, therefore, the relation between $F 8$ mutation and inhibitor risk could not be explained by FVIII:C level.

An unexpected high incidence of inhibitors occurred after 50 exposure days. Development of inhibitors later in life has been recently recognized in hemophilia A patients with prior extensive exposure to factor concentrates. ${ }^{17,19}$ The possibility of age-related immune dysregulation and late loss of tolerance may be particularly relevant to the nonsevere hemophilia cohort, as exposure to therapeutic factor VIII concentrates is distributed throughout life and often skewed to later decades for elective operative interventions. Current hypotheses suggest that this may be elicited by the clinical circumstances during exposure that trigger the immune system, such as intensive treatment with factor VIII concentrates for surgery. ${ }^{20,21}$ Although we adjusted for cumulative number of exposure days, the present analysis does not take potential clinical risk factors (such as factor VIII product type, intensity, and reason for treatment) into account. ${ }^{19-21}$ However, it is highly unlikely that these clinical risk factors have influenced the observed association between $F 8$ genotype and inhibitor development due to the principle of Mendelian randomization: inheritance of a certain genotype is "random" and will not be influenced by environmental determinants of inhibitor development. ${ }^{22}$

When interpreting the results of this study there are certain limitations that should be kept in mind. We obtained data from nonsevere patients that did receive treatment with factor VIII concentrates. Patients with a very mild bleeding phenotype or those exclusively treated with desmopressin were not included. As those who need treatment with factor VIII concentrates do not represent all nonsevere hemophilia A patients, the incidence of inhibitors in the total nonsevere hemophilia A population will be lower than reported in our study. The applicability of our results is also limited to white populations, as the study population was mainly white.

Only if new $F 8$ mutations would occur at a high rate, this could introduce confounding, as FVIII product type has also changed over time. However, the spontaneous mutation rate in nonsevere hemophilia is described to be very low $(5 \%){ }^{23}$ Therefore, we do not expect this to be an important confounder in our analysis.

Because family members who share the same $F 8$ mutation are more likely to share allelic variants of other genes (eg, immuneresponse genes [TNFA, IL-10 or CTLA-4]) that increase the risk for inhibitor development in severe hemophilia A, we cannot exclude that other inherited factors may also have contributed to the observed associations. $^{24-27}$

Theoretically there is one specific situation that may lead to a biased estimate of the effect of $F 8$ genotype on inhibitor risk that has been explained in an article by Sheehan et al. ${ }^{28}$ This could occur if there would be another gene that affects both inhibitor risk and is also linked to the $F 8$ mutation. In this case, the second gene may confound the association between $F 8$ mutation and disease. Currently, there are no data on a gene linked to $F 8$ that also causes inhibitors, but of course this is theoretically possible. The low incidence $(2.3 \%)$ of patients with a positive family history of inhibitors in our cohort is likely to be a function of challenges of data collection associating a particular individual with other family members (especially when they are treated in different centers) and emphasizes the need for decent family tree maintenance.

Despite the large number of patients included in our study, we acknowledge that our study has limited statistical power to calculate the inhibitor risk for most of the $F 8$ mutations, because these mutations only occur in a small number of patients or because the patients with these specific mutations have a limited number of exposure days. However, considering the extent of this study, it may never be duplicated or prospectively studied. Given that inhibitors were not regularly tested in all patients after a predefined regimen, we may have missed patients with asymptomatic or transient inhibitors, especially in the beginning of the observation period (1980-2000). Therefore, the inhibitor incidences in our study may be underestimated. To reduce the potential influence of interassay and interlaboratory variability on inhibitor classification, we used a clinically relevant inhibitor as the outcome measure. $^{29}$

Our study identifies a higher incidence of inhibitor development in nonsevere hemophilia than previously appreciated and has made 
A

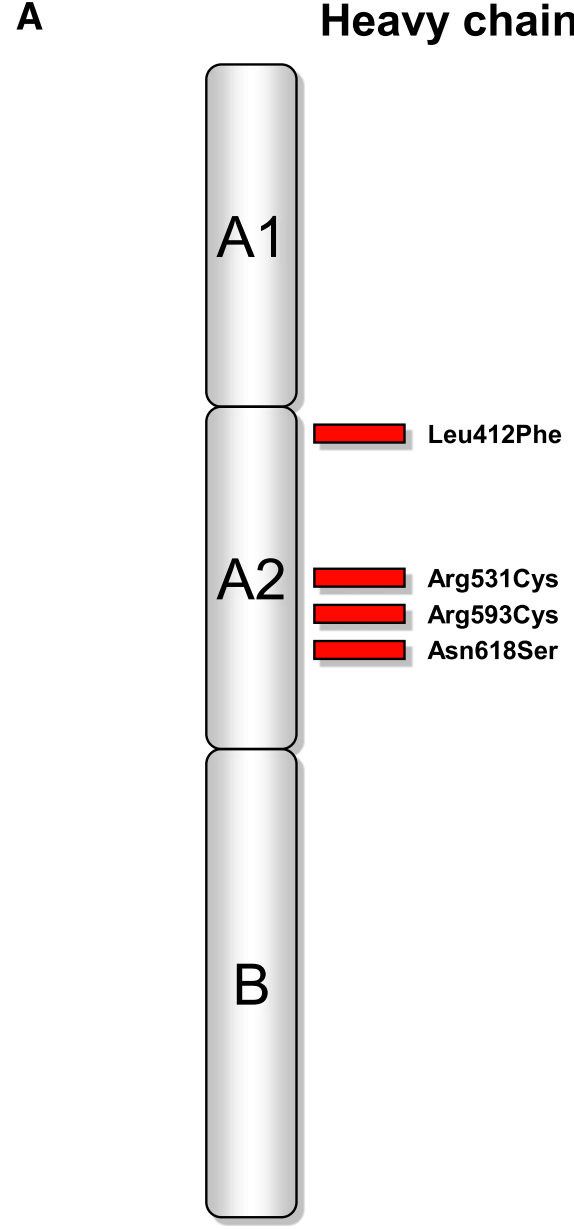

B

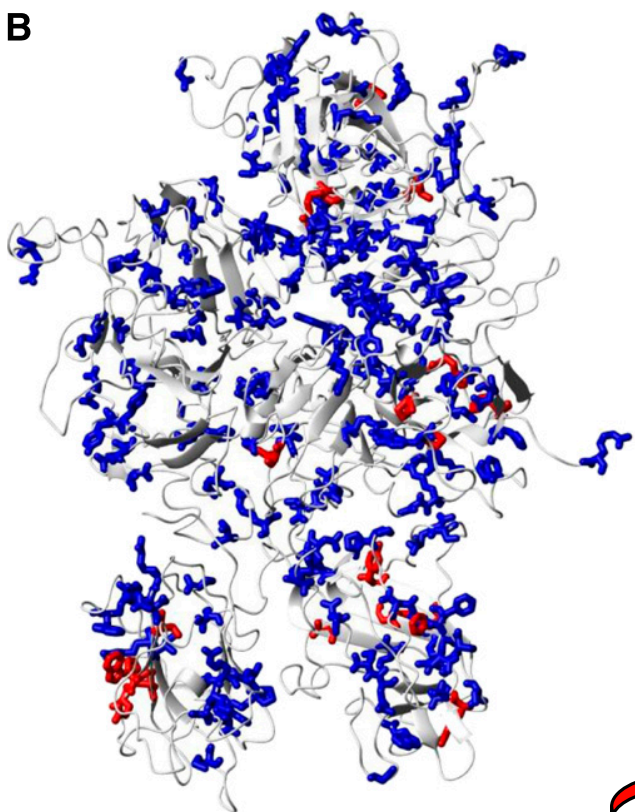

\section{Light chain}

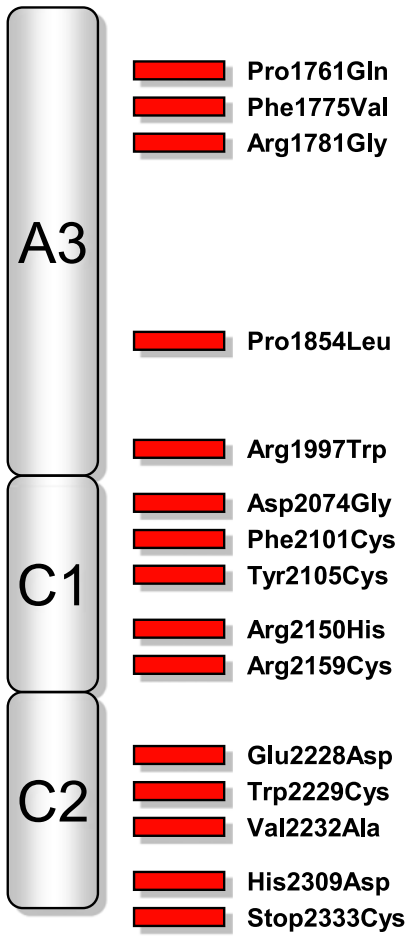

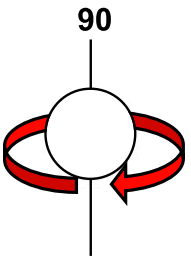

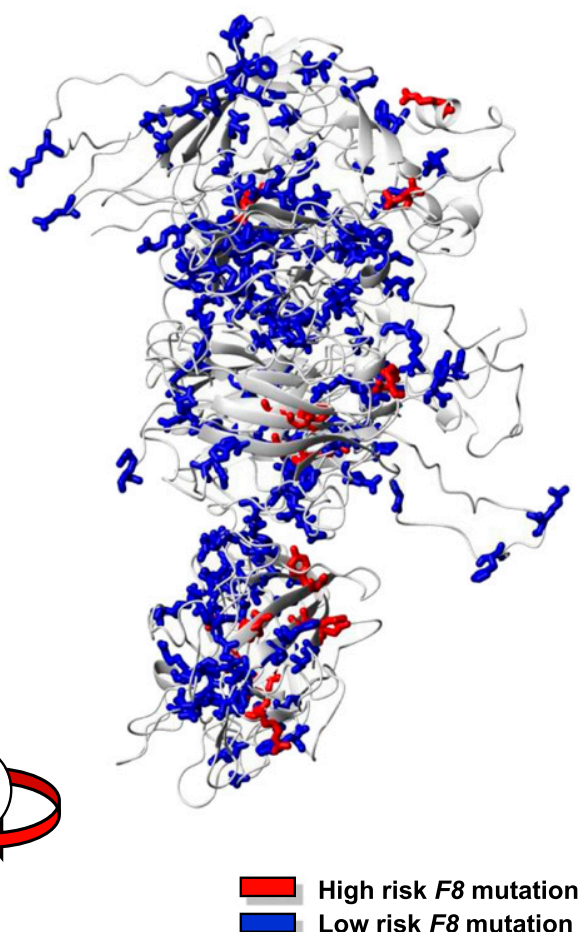

Figure 3. Distribution of $\mathbf{F} 8$ missense mutations associated with inhibitor development. (A) Two-dimensional and (B) three-dimensional structure of the factor VIII protein. 
Table 2. Inhibitor risk of the 19 F8 mutations in which inhibitor development occurred

\begin{tabular}{|c|c|c|c|c|c|c|c|c|}
\hline F8 mutation & $\begin{array}{l}\text { No. of } \\
\text { patients } \\
\mathrm{n}(\%)^{\star}\end{array}$ & $\begin{array}{c}\text { No. of patients } \\
\text { with inhibitor } \\
n(\%)\end{array}$ & $\begin{array}{l}\text { Baseline FVIII:C, } \\
\text { IU/dL median } \\
\text { (min-max) }\end{array}$ & $\begin{array}{c}\text { End of } \\
\text { follow-upt } \\
\text { (Cum no. ED) }\end{array}$ & $\begin{array}{c}\text { No. of patients } \\
\text { under observation } \\
\text { at } 20 \mathrm{ED}\end{array}$ & $\begin{array}{c}\text { Inhibitor risk at } \\
20 \mathrm{ED} \neq \\
\%(95 \% \mathrm{Cl})\end{array}$ & $\begin{array}{c}\text { No. of patients } \\
\text { under observation } \\
\text { at } 50 \mathrm{ED}\end{array}$ & $\begin{array}{c}\text { Inhibitor risk } \\
\text { at } 50 \mathrm{ED} \neq \\
\%(95 \% \mathrm{Cl})\end{array}$ \\
\hline \multicolumn{9}{|c|}{ Mutations in 10 or more patients } \\
\hline Arg531Cys & $35(3.2)$ & $1(2.9)$ & $8(2-37)$ & $>100$ & 25 & 0.0 & 16 & $0.0 \S$ \\
\hline Arg593Cysll & $106(9.5)$ & $12(11.3)$ & $17(5-32)$ & $>100$ & 35 & $9.1(2-16)$ & 16 & $18.3(7-30)$ \\
\hline Asn618Ser & $58(5.2)$ & $1(1.7)$ & $24(8-37)$ & $>100$ & 18 & $2.9(0-9)$ & 5 & $2.9(0-9)$ \\
\hline Asp2074Gly & $11(1.0)$ & $3(27.3)$ & $8(4-14)$ & $>100$ & 7 & $21.2(0-47)$ & 5 & $21.2(0-47)$ \\
\hline Arg2150His & $57(5.1)$ & $9(15.8)$ & $7(2-32)$ & $>100$ & 36 & $2.2(0-7)$ & 20 & $12.2(1-24)$ \\
\hline Arg2159Cys & $21(1.9)$ & $3(14.3)$ & $14(6-29)$ & $>100$ & 8 & $9.1(0-26)$ & 3 & $39.4(3-75)$ \\
\hline Trp2229Cys & $10(0.9)$ & $5(50.0)$ & $8(5-24)$ & $50-100$ & 4 & $41.7(5-78)$ & 3 & $41.7(5-78)$ \\
\hline \multicolumn{9}{|c|}{ Mutations in less than 10 patients } \\
\hline Leu412Phe & $5(0.4)$ & $1(20.0)$ & $8(3-14)$ & $>100$ & & & & \\
\hline Pro1761Gln & $2(0.2)$ & $1(50.0)$ & $6(5-6)$ & $>100$ & & & & \\
\hline Phe1775Val & $3(0.3)$ & $2(66.7)$ & $27(13-29)$ & $10-20$ & & & & \\
\hline Arg1781Gly & $4(0.4)$ & $1(25.0)$ & $8(6-16)$ & $50-100$ & & & & \\
\hline Pro1854Leu & $4(0.4)$ & $1(25.0)$ & $14(6-25)$ & $>100$ & & & & \\
\hline Arg1997Trp & $3(0.3)$ & $2(66.7)$ & $4(4-6)$ & $>100$ & & & & \\
\hline Phe2101Cys & $2(0.2)$ & $2(100)$ & $7(6-7)$ & $50-100$ & & & & \\
\hline Tyr2105Cys & $6(0.5)$ & $3(50.0)$ & $19(12-28)$ & $20-30$ & & & & \\
\hline Glu2228Asp & $3(0.3)$ & 1 (33.3) & 27 (16-36) & $10-20$ & & & & \\
\hline Val2232Ala & $1(0.1)$ & $1(100.0)$ & 15 & $30-50$ & & & & \\
\hline His2309Asp & $1(0.1)$ & $1(100.0)$ & 2 & $30-50$ & & & & \\
\hline Stop2333Cys & $1(0.1)$ & $1(100.0)$ & 11 & $5-10$ & & & & \\
\hline Total & $333(29.9)$ & $51(15.3)$ & & & & & & \\
\hline
\end{tabular}

the first step toward identification of high risk patients based on their F8 genotype. As there are currently no proven strategies to prevent inhibitor development in nonsevere hemophilia patients, further research to develop preventive strategies is urgently needed. In the absence of these strategies, we propose that desmopressin response should be tested in all nonsevere hemophilia A patients to optimize the use of desmopressin in adequate responders as an effective and safe alternative for factor VIII concentrates, without the risk of inhibitor development. ${ }^{30}$ This is relevant because it appears that at least some high-risk mutations respond well to desmopressin administration. ${ }^{31}$ Our study also highlights the potential of $F 8$ genotyping to estimate individualized risks of inhibitor formation for those mutations with sufficient data. It can inform the patientdoctor consultation, contributing to the decision whether to proceed with an elective intervention and how to manage it, and may therefore be of immediate clinical relevance. Moreover, the results of our study contribute to our knowledge of alloreactivity in response to an intravenous protein therapeutic.

\section{Acknowledgments}

The authors thank Prof J. Voorberg, S. Meijer (Sanquin, Amsterdam, The Netherlands), Prof J.C.M. Meijers, Prof S. Middeldorp, and Prof M.M. Levi (Academic Medical Center, Amsterdam, The Netherlands) for their critical review and suggestions to improve the manuscript.

This work was supported by a grant from The Netherlands Organisation for Health Research and Development (ZonMw grant 40-00703-98-8570) (C.L.E and K.F.), and an unrestricted research grant from CSL Behring (M.P. and K.F).

The sponsors had no role in the choice of members of the steering committee and the participating centers nor the design and conduct of the study; collection, management, analysis, and interpretation of the data; and preparation, review, and approval of the manuscript.

\section{Authorship}

Contribution: C.L.E. and A.S.v.V. interpreted the data; C.L.E. analyzed the data and wrote the manuscript; A.S.v.V. edited the final version of the manuscript; J.G.B. and K.F. designed the study; J.G.B. supervised the interpretation and statistical analysis of the data; K.F. supervised the study, wrote the protocol, and wrote and edited the manuscript; and C.L.E., A.S.v.V., M.P., J.A., P.P.B., G.C., M.H.C., N.D., C.E.-E., K.H., D.P.H., C.R.M.H., S.H., W.L.v.H., C.H., M.H., V.J.-Y., R.D.K., R.K., B.A.P.L.-v.G., F.W.G.L., R.L., A.M., C.M., E.M.-B., M.G.M., S.M., K.M., M.Mi., M.Mo., M.N., J.O., K.P., P.P., H.P., S.E.R.-P., E.S., P.S., F.J.S., B.S., A.T., T.T.Y., P.W.K., J.G.v.d.B., and K.F. collected data or supervised data collection, and reviewed and approved the final version of the manuscript.

Conflict-of-interest disclosure: C.L.E. has received an unrestricted grant from The Netherlands Organisation for Health Research and Development (ZonMW) and has given lectures at educational symposiums organised by Novo Nordisk and Baxter. J.A. has received honorarium for advisory boards and lectures from Pfizer, CSL Behring, SOBI, Novo Nordisk, Baxter, and Bayer, and has received grants from Baxter, Grifols, and Bayer. 
P.P.B. has received payment for consultancy meetings with Novartis, has participated in advisory boards for Novartis, and has received travel grants from Baxter, Bayer, Novartis, Pfizer, and CSL Behring. G.C. has received payment for lectures from Baxter, Pfizer, CSL Behring, Novo Nordisk, and Kedrion. D.P.H. has received the Bayer Early Career Investigator Award, and has received payment for lectures from Baxter, Bayer, and Octapharma. C.R.M.H. is board member of the Baxter recombinant IX Data Safety Monitoring Board, has received payment for consultancy meetings with Baxter, Pfizer, Inspiration, and Novo Nordisk, has received unrestricted grant supporting research from Baxter, Pfizer, Bayer, and CSL Behring, has received payment for lectures from Baxter, Bayer, Pfizer, Novo Nordisk, CSL Behring, Grifols, and LFB, and has received payment to travel to scientific meetings. S.H. has received payment for lectures from Novo Nordisk, and has received payment for travel expenses from Bayer. W.L.v.H. is co-owner of spin off HaemoMagum BV, has received several unrestricted educational grants from Baxter, CSL Behring, and Novo Nordisk, and has received payment for lectures from Roche Diagnostics. V.J.-Y. is a member of the European Hemophilia Treatment and Standardisation Board sponsored by Baxter, has previously received payment for consultancy meetings with Pfizer, Grifols, Novo Nordisk, and Bayer, has received grants from Octapharma and Novo Nordisk, and has received payment for lectures from Baxter, Novo Nordisk, Pfizer, Grifols, and Octapharma. R.K. has participated in advisory boards for Baxter, Bayer, CSL Behring, Pfizer, and Novo Nordisk, and has received payment for lectures from Bayer, Baxter, CSL Behring, Novo Nordisk, SOBI, and Pfizer. R.L. has received payment for consultancy meetings with Baxter and Bayer, has received payment for lectures from Pfizer and Bayer, and has received payment for development of educational presentations by Pfizer and Bayer. C.M. has received an unrestricted grant from CSL Behring, has received payment for lectures from CSL Behring, Wyeth, Bayer, Baxter, and Biotest, and has received travel expenses paid by CSL Behring, Wyeth/Pfizer, Bayer, Baxter, and Biotest. M.G.M. has received payment for consultancy meetings with Baxter, Novo Nordisk, Bayer, Pfizer, Amgen, Glaxo, and Shire, and has received payment for lectures from Baxter, Novo Nordisk, Bayer, Pfizer, Amgen,
Glaxo, and Shire. M.M. has participated in advisory boards for Novo Nordisk, Pfizer, and CSL Behring, has received payment for consultancy meetings with Bayer and Biotest, and has received payment for lectures from Bayer and Novo Nordisk. K.P. has participated in advisory boards for Pfizer, Baxter, and Bayer, has received payment for lectures by Novo Nordisk, has received travel grants from Baxter, Bayer, and CSL Behring, and has an unrestricted chair to the University for research in Haemophilia paid for by Pfizer, Baxter, and CSL Behring. P.P. is a board member of the Medical Advisory Board Pfizer, has received payment for lectures from Bayer, Baxter, and Pfizer, and has received travel expenses for hemophilia meetings. S.E.R.-P. has received the Bayer Haemophilia Clinical Training Award, has received payment for lectures from Bayer and Novo Nordisk, and has received payment for travel expenses for participation in various congresses. E.S. has participated in advisory boards for Bayer, Pfizer, Novo Nordisk, and CSL Behring, has received payment for consultancy meetings with Grifols and Kedrion, has received grants from Pfizer and Novo Nordisk, has received payment for lectures from Biotest and Octapharma, and has received travel expenses paid by Baxter. P.S. is a board member of Bayer International Advisory Board, has received payment for consultancy meetings with Baxter, Novo Nordisk, and Pfizer and has received payment for lectures from Bayer, Baxter, and Amgen. J.G.v.d.B. has received payment for consultancy meetings with Bayer and Wyeth, has received grants from Bayer Schering Pharma, Baxter, CSL Behring, Novo Nordisk, and Wyeth, and has received payment for lectures from Bayer. K.F. is a member of the European Hemophilia Treatment and Standardisation Board sponsored by Baxter, has received unrestricted research grants from CSL Behring, Pfizer, and Bayer, and has given lectures at educational symposiums organised by Pfizer and Bayer. The remaining authors declare no competing financial interests.

A complete list of the members of the INSIGHT Study Group appears in the online data supplement.

Correspondence: Karin Fijnvandraat, Department of Pediatric Hematology, Emma Children's Hospital, Room H7-270 Academic Medical Center, Meibergdreef 9, 1105 AZ, Amsterdam, The Netherlands; e-mail: c.j.fijnvandraat@amc.uva.nl.

\section{References}

1. Wight $\mathrm{J}$, Paisley $\mathrm{S}$. The epidemiology of inhibitors in haemophilia $A$ : a systematic review. Haemophilia. 2003;9(4):418-435.

2. Fijnvandraat $\mathrm{K}$, Cnossen $\mathrm{MH}$, Leebeek FW Peters M. Diagnosis and management of haemophilia. BMJ. 2012;344:e2707.

3. Di Minno MN, Di Minno G, Di Capua M, Cerbone AM, Coppola A. Cost of care of haemophilia with inhibitors. Haemophilia. 2010;16(1):e190-e201.

4. Hay CR, Ludlam CA, Colvin BT, et al; UK Haemophilia Centre Directors Organisation. Factor VIII inhibitors in mild and moderateseverity haemophilia A. Thromb Haemost. 1998 79(4):762-766.

5. Boekhorst J, Lari GR, D'Oiron R, et al. Factor VIII genotype and inhibitor development in patients with haemophilia $A$ : highest risk in patients with splice site mutations. Haemophilia. 2008;14(4): 729-735.

6. Oldenburg J, El-Maarri O, Schwaab R. Inhibitor development in correlation to factor VIII genotypes. Haemophilia. 2002;8(Suppl 2):23-29.

7. Gouw SC, van den Berg HM, Oldenburg J, et al. F8 gene mutation type and inhibitor development in patients with severe hemophilia $A$ : systematic review and meta-analysis. Blood. 2012;119(12): 2922-2934.
8. d'Oiron R, Pipe SW, Jacquemin M. Mild/moderate haemophilia $A$ : new insights into molecular mechanisms and inhibitor development. Haemophilia. 2008;14(Suppl 3):138-146.

9. Kemball-Cook G. The Haemophilia A Mutation, Structure, Test and Resource Site. http://www. hadb.org.uk/. Accessed April 15, 2013.

10. Centers for Disease Control and Prevention. CHAMP: CDC Hemophilia A Mutation Project. http://www.cdc.gov/ncbddd/hemophilia/champs. html. Accessed April 15, 2013.

11. Vidal F, Farssac E, Altisent C, Puig L, Gallardo D. Rapid hemophilia A molecular diagnosis by a simple DNA sequencing procedure: identification of 14 novel mutations. Thromb Haemost. 2001; 85(4):580-583.

12. Genomic Disorders Research Centre. HGVS: Human Genome Variation Society. http://www. hgvs.org. Accessed February 5, 2013.

13. Kasper CK, Aledort L, Aronson D, et al. Proceedings: A more uniform measurement of factor VIII inhibitors. Thromb Diath Haemorrh. 1975;34(2):612.

14. Verbruggen $B$, Novakova I, Wessels $H$, Boezeman J, van den Berg M, MauserBunschoten $\mathrm{E}$. The Nijmegen modification of the Bethesda assay for factor VIII:C inhibitors: improved specificity and reliability. Thromb Haemost. 1995;73(2):247-251.

15. Harrel FE. Missing Data: With Applications to Linear Models, Logistic Regression, and Survival Analysis. New York, NY: Springer; 2001.

16. Collins PW, Chalmers E, Hart DP, et al. Diagnosis and treatment of factor VIII and IX inhibitors in congenital haemophilia: (4th edition). UK Haemophilia Centre Doctors Organization. $\mathrm{Br} \mathrm{J}$ Haematol. 2013;160(2):153-70.

17. Hay CR, Palmer B, Chalmers E, et al; United Kingdom Haemophilia Centre Doctors' Organisation (UKHCDO). Incidence of factor VIII inhibitors throughout life in severe hemophilia $A$ in the United Kingdom. Blood. 2011;117(23): 6367-6370.

18. Schwaab R, Pavlova A, Albert T, Caspers M, Oldenburg J. Significance of F8 missense mutations with respect to inhibitor formation. Thromb Haemost. 2013;109(3):464-470.

19. Kempton $\mathrm{CL}$, Soucie JM, Miller $\mathrm{CH}$ et al. In nonsevere hemophilia $A$ the risk of inhibitor after intensive factor treatment is greater in older patients: a case-control study. J Thromb Haemost. 2010;8(10):2224-2231.

20. Eckhardt $\mathrm{CL}$, Menke LA, van Ommen $\mathrm{CH}$, et al. Intensive peri-operative use of factor VIII and the 
Arg593 - > Cys mutation are risk factors for inhibitor development in mild/moderate hemophilia A. J Thromb Haemost. 2009;7(6):930-937.

21. Eckhardt CL, van der Bom JG, van der Naald M, Peters M, Kamphuisen PW, Fijnvandraat K. Surgery and inhibitor development in hemophilia A: a systematic review. J Thromb Haemost. 2011; 9(10):1948-1958.

22. Smith GD, Ebrahim $S$. Mendelian randomization: prospects, potentials, and limitations. Int $J$ Epidemiol. 2004;33(1):30-42.

23. Strauss HS. The perpetuation of hemophilia by mutation. Pediatrics. 1967;39(2):186-193.

24. Astermark J, Oldenburg J, Carlson J, Pavlova A, Kavakli K, Berntorp E, Lefvert AK. Polymorphisms in the TNFA gene and the risk of inhibitor development in patients with hemophilia A. Blood. 2006;108(12):3739-3745.
25. Astermark J, Oldenburg J, Pavlova A, Berntorp E, Lefvert AK; MIBS Study Group. Polymorphisms in the IL 10 but not in the IL1beta and IL4 genes are associated with inhibitor development in patients with hemophilia A. Blood. 2006;107(8): 3167-3172.

26. Astermark J, Wang X, Oldenburg J, Berntorp E, Lefvert AK; MIBS Study Group. Polymorphisms in the CTLA-4 gene and inhibitor development in patients with severe hemophilia A. J Thromb Haemost. 2007;5(2): 263-265.

27. Astermark J, Berntorp E, White GC, Kroner BL; MIBS Study Group. The Malmö International Brother Study (MIBS): further support for genetic predisposition to inhibitor development in hemophilia patients. Haemophilia. 2001;7(3): 267-272.
28. Sheehan NA, Didelez V, Burton PR, Tobin MD. Mendelian randomisation and causal inference in observational epidemiology. PLoS Med. 2008; 5(8):e177.

29. Meijer $P$, Verbruggen $B$. The between-laboratory variation of factor VIII inhibitor testing: the experience of the external quality assessment program of the ECAT foundation. Semin Thromb Hemost. 2009;35(8):786-793.

30. Mannucci PM. Desmopressin (DDAVP) in the treatment of bleeding disorders: the first twenty years. Haemophilia. 2000;6(Suppl 1):60-67.

31. Castaman G, Mancuso ME, Giacomelli SH, Tosetto A, Santagostino E, Mannucci PM, Rodeghiero F. Molecular and phenotypic determinants of the response to desmopressin in adult patients with mild hemophilia A. J Thromb Haemost. 2009;7(11):1824-1831. 


\section{( ) blood}

2013 122: 1954-1962

doi:10.1182/blood-2013-02-483263 originally published online August 7, 2013

\section{Factor VIII gene (F8) mutation and risk of inhibitor development in nonsevere hemophilia A}

Corien L. Eckhardt, Alice S. van Velzen, Marjolein Peters, Jan Astermark, Paul P. Brons, Giancarlo Castaman, Marjon H. Cnossen, Natasja Dors, Carmen Escuriola-Ettingshausen, Karly Hamulyak, Daniel P. Hart, Charles R. M. Hay, Saturnino Haya, Waander L. van Heerde, Cedric Hermans, Margareta Holmström, Victor Jimenez-Yuste, Russell D. Keenan, Robert Klamroth, Britta A. P. Laros-van Gorkom, Frank W. G. Leebeek, Ri Liesner, Anne Mäkipernaa, Christoph Male, Evelien Mauser-Bunschoten, Maria G. Mazzucconi, Simon McRae, Karina Meijer, Michael Mitchell, Massimo Morfini, Marten Nijziel, Johannes Oldenburg, Kathelijne Peerlinck, Pia Petrini, Helena Platokouki, Sylvia E. Reitter-Pfoertner, Elena Santagostino, Piercarla Schinco, Frans J. Smiers, Berthold Siegmund, Annarita Tagliaferri, Thynn T. Yee, Pieter Willem Kamphuisen, Johanna G. van der Bom and Karin Fijnvandraat

Updated information and services can be found at:

http://www.bloodjournal.org/content/122/11/1954.full.html

Articles on similar topics can be found in the following Blood collections

Free Research Articles (3297 articles)

Pediatric Hematology (413 articles)

Thrombosis and Hemostasis (882 articles)

Information about reproducing this article in parts or in its entirety may be found online at:

http://www.bloodjournal.org/site/misc/rights.xhtml\#repub_requests

Information about ordering reprints may be found online at:

http://www.bloodjournal.org/site/misc/rights.xhtml\#reprints

Information about subscriptions and ASH membership may be found online at:

http://www.bloodjournal.org/site/subscriptions/index.xhtml

Blood (print ISSN 0006-4971, online ISSN 1528-0020), is published weekly by the American Society of Hematology, 2021 L St, NW, Suite 900, Washington DC 20036.

Copyright 2011 by The American Society of Hematology; all rights reserved. 